\title{
Tailoring self-assembled monolayers at the electrochemical interface ${ }^{\dagger}$
}

\author{
S VARATHARAJAN, SHEELA BERCHMANS and V YEGNARAMAN* \\ Central Electrochemical Research Institute, Karaikudi 630006 \\ e-mail: vyegna@gmail.com
}

\begin{abstract}
The main focus of this review is to illustrate the amenability of self-assembled monolayers (SAMs) for functionalisation with different receptors, catalytic materials, biomolecules, enzymes, antigen-antibody, etc for various applications. The review discusses initially about the preparation and characterization of SAMs and tailoring of SAMs by incorporation of suitable recognition elements. A description of how the molecular recognition is achieved through forces like electrostatic, covalent and host-guest interactions is included in the review.
\end{abstract}

Keywords. Self-assembled monolayers; molecular recognition; electrostatic interactions; host-guest interactions; covalent interactions.

\section{Introduction}

Studies on organized monolayers dates back to the ingenious work of Agnes Pockels who at the end of the 19th century designed the first apparatus to study films at the air-water interface. ${ }^{1}$ Langmuir and Blodgett ${ }^{2}$ developed methods for transfer of layers on solid support and this started the wave of research on surface science in the area of organized monolayers. Zisman ${ }^{3}$ and co-workers in 1940s performed the first systematic study of self-assembled monolayers (SAMs). He noticed that a clean platinum object immersed, and then carefully withdrawn from the solution of an amphiphile becomes hydrophobic and concluded that amphiphile adsorbs on the surface to produce monolayers. SAM can be defined as a molecular assembly that is formed spontaneously by the immersion of an appropriate substrate into a solution of an active surfactant in a suitable solvent. ${ }^{4}$ The driving force for self-assembly is usually the specific interactions between the head group of the surfactant and the surface of the substrate. Most surfactants used for forming SAMs consist of three distinctive parts: surface active head group which binds strongly to the surface, end (or tail) group which is located at the monolayer's surface and normally determines the interfacial properties of the assembly and the alkane chain which facilitates the packing of the molecules in the

\footnotetext{
${ }^{\dagger}$ Dedicated to the memory of the late Professor S K Rangarajan *For correspondence
}

monolayers and serves as a linker between the head and the end groups. Figure 1 illustrates formation of SAMs and shows some intermolecular interactions important for self assembly.

Many examples of surface-surfactant interactions, which promote self-assembly are known. Apart from gold-thiol monolayers, which are formed due to formation of the strong S-Au bond, other commonly studied monolayers include alkyl trichlorosilane layers on hydroxylated surfaces (such as $\mathrm{SiO}_{2}$ ), fatty acids on metal oxide surfaces and alkyl phosphonates on zirconium and selenols on silver. ${ }^{5}$

Nuzzo and Allara discovered the ability of disulfides and thiols to self-assemble on gold surfaces ${ }^{6}$ in 1983 and in the 1990s these films have probably become the most studied monolayers worldwide. Both

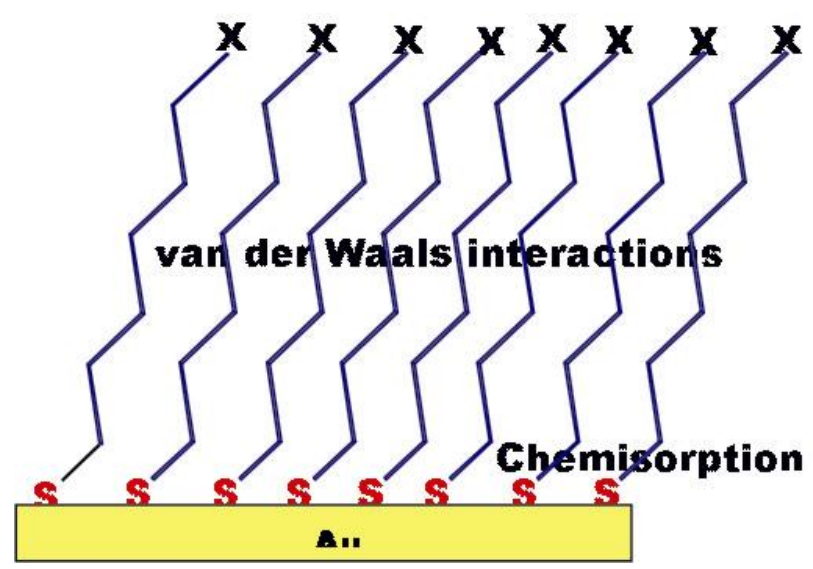

Figure 1. Schematic diagram of a SAM on gold substrate. 
thiol and disulfide monolayers on gold are stable under normal laboratory conditions, and no scrupulous precautions (such as exceptional purity of the reagents or clean room conditions) are required to produce monolayers. Thiols and disulphides are compatible with most organic functional groups and this makes it possible to incorporate a huge variety of terminal functionalities in gold-thiol monolayers. The synthetic chemistry of thiols and disulphides is well established and in most cases they are very simple. Simple thiols and the precursors for more sophisticated adsorbates are commercially available and cheap. Furthermore, Au surface, is conveniently prepared by thermal evapouration on supports such as silicon or glass slides.

\section{Formation of SAM}

SAMs of alkane thiols on Au are formed by adsorption from solution (or vapour) of a long chain alkane thiol $\left(\mathrm{X}-\left(\mathrm{CH}_{2}\right)_{n}-\mathrm{SH} ; n=11-18\right)$ to Au surface and is represented by

$$
\mathrm{RSH}+\mathrm{Au}_{n}^{0} \rightarrow \mathrm{RS}^{-}-\mathrm{Au}^{+} \mathrm{Au}_{n-1}^{0}+1 / 2 \mathrm{H}_{2} .
$$

The gold-thiol SAM formation is simple and has been investigated extensively. Further, the reaction kinetics of gold with oxygen is extremely slow and hence gold substrates can be handled in ambient condition in air. The affinity of thiols for gold is so strong that it replaces most other adsorbates and forms stable monolayer. ${ }^{7}$ The structure of SAM depends on the morphology of the metal. Au (111) is mostly employed for the formation of monolayer. In a typical procedure, the gold surface is first pretreated with piranha solution, ${ }^{8}$ successively rinsed with deionized water and absolute alcohol and then immersed in a $1 \mathrm{mM}$ solution of appropriate thiol in high purity ethanol for 16 to $24 \mathrm{~h}$ at room temperature. Subsequently, the surface is withdrawn and rinsed with ethanol and dried. The solvent, widely used for monolayer deposition is ethanol. Other solvents such as water, hexane, toluene, tetrahydrofuran (THF), dichloromethane, acetonitrile and DMF can also be used without affecting the structure of resultant monolayer. Monolayers prepared by using thiols with long alkane chain (hexadecane) were shown to hamper the formation of high quality layers. To ensure that the monolayer is not contaminated by co-adsorption of impurities, high purity solvent should be used. An uncontaminated gold surface is important for the formation of SAM, however it is not essential. The high affinity for thiol moiety for gold even displaces contaminants. ${ }^{9}$ Alkanethiols from dilute solution form a densely packed monolayer in less than $1 \mathrm{~h}$. The adsorption time is independent of the chain length, but depends on the concentration of the thiol. Generally high concentration leads to shorter adsorption time. In the case of mixed SAMs, the mole ratio of a mixture of thiols in solution results in the same ratio. The two components do not phase segregate into islands. ${ }^{10}$ This interesting feature is exploited to immobilize biomolecules in such a manner that steric hindrance between these molecules and their binding partners is avoided. The multilayer formation is not possible for alkanethiols $(n>5)$. The multilayer is observed only after 6 days of immersion. The assembling kinetics of a monolayer is biphasic: a re-crystallization process follows the slow diffusion-controlled adsorption.

\section{Characterization of SAM}

\subsection{Infra-red spectroscopy}

Much informative data on the chemical composition of gold-thiol monolayer can be obtained from grazing angle FTIR spectroscopy. ${ }^{11-13}$ Reflective IR spectroscopy measures the amount of light absorbed by the thin film. Spectrum obtained is the difference between s-and p-polarized light which is sensitive to the SAM. The effect of change in chain length of the SAM could be studied by this method. The IR beam is reflected at a small grazing angle from the monolayer surface and the intensity of the reflected beam is recorded. Only the transition dipole having a component perpendicular to the metal surface will be IR active. If the chains are perpendicular to the metal surface, then asymmetric $-\mathrm{CH}_{2}$ and symmetric $-\mathrm{CH}_{2}$ vibrations would be parallel to the plane of the surface and will be absent in spectrum. This method makes it possible to directly obtain the IR spectrum of the monolayer. This is very helpful to find the orientation of the monolayer.

The order of alkyl chains in SAM could be studied by IR spectroscopy. Peaks at $2918 \mathrm{~cm}^{-1}$ and $2846 \mathrm{~cm}^{-1}$ are characteristic of ordered alkyl chain assembly. Decreased order is observed when the chain length is less than 11 , and when the length is less than 6 , the assembly is disordered.

\subsection{Surface enhanced Raman spectroscopy (SERS)}

SERS has been used to obtain useful information about the chemical structure of monolayer. This 
method requires a careful preparation of a roughened surface. It is very sensitive to the functionalities closed to the metal surface. ${ }^{14}$ This technique is based on the enhancement of Raman signals molecules adsorbed on rough metal surface. A distance dependence study of the surface enhanced Raman (SER) effect has been performed using alkanethiol $\left[\mathrm{CH}_{3}-\left(\mathrm{CH}_{2}\right)_{n} \mathrm{SH}\right] \mathrm{SAMs}$ on top of rough silver surfaces by Giuseppe Compagnini et $a l^{15}$. They found decrease of the enhancement signal of the $\mathrm{CH}_{3}$ heads by a factor of 2 , increasing the $\mathrm{CH}_{3}$ surface distance from 0.8 to $2.5 \mathrm{~nm}(n=5-17)$.

Structural differences in SAMs of antharquinone derivatives on Ag and Au electrodes were studied by in situ SER spectroscopy. ${ }^{16}$ SER spectrum of one of the anthraquinone derivatives (2-AQS), in which a perpendicular orientation of the anthraquinone plane relative to $\mathrm{Au}$ surface shows enhanced spectrum independent of the applied potential. Another anthraquinone derivative, with a flat orientation gives no clear spectra.

SERS titration has been used to monitor the surface charge state of the self-assembled monolayer.

\subsection{X-ray photoelectron spectroscopy}

In X-ray photoelectron spectroscopy (XPS), the core electrons are knocked out and the binding energy of the ejected electrons helps to find out the composition of the monolayer. The response from the elements buried at different levels below the surface depends on take-off angle. At lower take-off angles, the ejection of photoelectrons from the atoms at the top layer will be much more as compared to those from the deeper layer. This study of XPS response as a function of the take-off angle, allows one to calculate how deeply certain elements are positioned with respect to the monolayer surface and thus helps to find the composition. NEXAFS (Near-edge X-ray absorption fine structure spectroscopy), TOF-SIMS (Time of flight secondary ion mass spectroscopy) are also used to find the elemental composition of monolayer.

\subsection{Contact angle measurement}

The contact angle between monolayer surface and liquid provides structural information. ${ }^{17}$ Comparison of contact angles of polar and non-polar liquids provides 3-D structural information about the monolayer. High contact angles of water and hexadecane on methyl-terminated thiol monolayers and the low contact angles on carboxylic acid and alcoholterminated monolayers indicate that the surface of monolayers consists of densely packed arrays of the tail groups of the thiol. For well-formed monolayers, advanced contact angles are close to 45 degrees.

\subsection{Ellipsometry}

It is a well-known, non-destructive, precise and accurate technique used for measuring the thickness of the film, optical properties and modelling of surface roughness. In the case of monolayers, it helps to determine whether single or multiple layers assembled on the surface. In ellipsometry, the light is shined, and polarized by passing through a linear polarizer, then elliptically polarized by passing through a compensator. The light that hits the sample is reflected and is linearly polarized. Analyzer detects the change of polarization. The detector processes the data and compares with computerized optical models to give the thickness and refractive index of the SAM.

\subsection{Scanning probe microscopy}

Scanning probe microscopy studies have been used to characterize the structure of the monolayer at the molecular level and also to understand the nature of the adsorbed film. AFM is able to offer the local structure information at atomic resolution by sensing the force difference between a small sharp tip and surface, which corresponded to the ending of the compliant cantilever, the tip attached to and is translated into the laser signal change of a position to photodiode.

In STM, the tunnelling of electron between the tip and surface is used to image the surface. STM helps to study the overlayer structure of adsorbates on metal surface. The presence of pinholes could be seen in STM. Formation of large ordered domains in benzene thiol SAMs on $\mathrm{Au}(111)$ was observed by STM. ${ }^{18}$ The kinetics of monolayer adsorption is studied by piezoelectric oscillators such as Quartz Crystal Microbalance (QCM), which can detect mass changes of the order of nanograms.

\subsection{Electrochemical characterization}

3.7a Capacitance measurement: Depending on the alkyl chain length of the SAMs, the electrontransfer resistance increased and the capacitance 
decreased. A good linear relationship was found between inverse capacitance and chain length for carboxyl-terminated SAMs. ${ }^{19}$ Monolayers can be effectively adsorbed and desorbed by electrochemical means. Monolayer surface coverage can be determined by capacitance measurements. By measuring the charge $(Q)$ needed to desorb from a monolayer, the surface coverage can be calculated by $\Gamma=Q / n F A$, where $\mathrm{n}$ is number of electron transfer involved in the process, $F$ is the Faraday constant and $A$ is the geometric area of electrode surface. The values of $Q$ are determined by integration of the area under the $i-E$ curves (obtained in $1 \mathrm{M} \mathrm{HClO}_{4}$ ) after compensating for charging current. ${ }^{20} \mathrm{~A}$ pinhole free monolayer acts as an ideal capacitor. A SAM covered electrode, compared to a bare one, demonstrates a strong decrease in capacity.

3.7b Blocking of electron transfer kinetics: With the decrease in the current for the SAM-covered electrode, one can estimate the pinhole area. Porter et al have estimated the maximum pinhole radius as $8 \mu \mathrm{m}$ and a fractional uncovered area of one percent or less. ${ }^{21}$ An extreme decrease in the faradaic current of a redox couple is indicative of a hydrophobic SAM. A small residual current is a strong indication of the presence of pinholes. With a view to analyse the structural organization of SAMs, the solution phase electron transfer across carboxylic acid terminated thiols was studied ${ }^{22}$ in our laboratory with the help of the two standard redox systems $\left[\mathrm{Fe}(\mathrm{CN})_{6}\right]^{4}$ ${ }^{13-}$ and $\left[\mathrm{Ru}\left(\mathrm{NH}_{3}\right)_{6}\right]^{2+/ 3+}$. The reversible electron transfer kinetics observed in the case of $\left[\mathrm{Ru}\left(\mathrm{NH}_{3}\right)_{6}\right]^{2+/ 3+}$ and $\left.\mathrm{Fe}(\mathrm{CN})_{6}\right]^{4 / 3-}$ on a bare electrode slows down in the presence of SAM, which is indicated by the increase in peak separation and decrease in peak currents. The blocking of ET kinetics is found to increase with increase in chain length and ET is completely blocked in the case of MUA. The voltammetric response can be correlated to the nature of pinholes/defects present in the monolayer. If the pinholes formed in the monolayer are very small and widely separated relative to the diffusion layer thickness, sigmoidal shaped voltammograms will be obtained showing the characteristics of a nanoelectrode ensemble at which the radial diffusion layer developed at individual nanoelectrodes are isolated from each other. Large pinholes or closely packed small pinholes will result in peak-shaped voltammograms similar to those observed on a macroelectrode. In the case of long chain alkane thiols, the monolayers are highly ordered and are devoid of electrochemically measurable pinholes. The rate of electron transfer at defect-free domains will be less than the rate at areas having defects (including pinholes and collapsed sites). In the case of defect-free domains electron tunneling takes place. Our experimental results with the two standard redox systems $\left[\mathrm{Fe}(\mathrm{CN})_{6}\right]^{4-/ 3-}$ and $\left[\mathrm{Ru}\left(\mathrm{NH}_{3}\right) 6\right]^{2+/ 3+}$ reveal that peakshaped voltammograms are obtained in most of the cases. Hence we are dealing with situations of large pinholes or closely packed small pinholes, which have given rise to peak-shaped voltammograms, similar to the case of macroscopic electrode. In the case of MUA complete blocking of ET kinetics is observed. This shows that the SAM is devoid of electrochemically measurable pinholes and consists of defect-free domains. Tunnelling current decreases exponentially with increase of monolayer thickness. The thickness of the SAM could also be measured by applying Marcus equation, $I=I_{0} e^{-\beta d}$ where $I=$ current on SAM electrode, $I_{0}=$ current on bare electrode, $d$ is the thickness of SAM and $\beta$ is a constant.

The apparent effective thickness of alkanethiol SAMs is measured by impedance spectroscopy using different concentrations of $\mathrm{Fe}(\mathrm{CN})_{6}^{3-}$ by Xiaoli Cui et $a l{ }^{23}$ They showed that the complex-plane impedance plots for SAMs in solution of various concentrations of $\mathrm{Fe}(\mathrm{CN})_{6}^{3-} / \mathrm{Fe}(\mathrm{CN})_{6}^{4-}$ exhibit arc shapes. The apparent resistance $\left(R_{2}\right)$, obtained from the simulation, decreased with increasing concentration of the redox couples. From that the apparent effective thickness of the SAMs is determined.

\section{Applications of SAMs}

Surface modification of metal electrodes is carried out by taking into consideration the potential applications and desired surface properties. Among the surface-modification techniques, self-assembly (SA) technique is one of the most common approaches for modification of metal electrodes. Because of the ease of preparation, SAMs can be suitably functionalized by changing the tail group. SA is a kind of 'bottom up' technique, which provides advantages regarding the surface modification. ${ }^{24}$ It leads to a structure which is at, or close to, thermodynamic equilibrium, and thus tends to self-healing/defect rejection and leads to a closely packed, well-ordered, and stable configuration on the surface. ${ }^{25}$ The SAMs provide the needed design flexibility, both at the in- 
dividual molecular and at the material levels, and offer a tool for fabrication of specific interfaces or complex surfaces on the two-dimensional or threedimensional substrates. The novelty of using the SAMs stems from their ability to be further modified into chemically or biologically reactive surface layers (via covalent coupling of different materials to functional end group). This use of the SAMs for anchoring various functionalities can impart specific interactions essential for various sensing applications.

A variety of molecular assemblies have been tailored with SAMs, which include proteins, enzymes, antigens, antibodies, lipids, DNA, small biomolecules, metal ions, molecular assemblies containing nanocavitites, viz. cyclodextrins, calixarenes, carceplexes and so on. The applications include electron transfer kinetics, sensors, catalysis, etc. In this review, we discuss the different protocols that are reported in the literature to functionalize SAM modified interfaces for different types of molecular recognition.

\subsection{Molecular recognition}

Molecular recognition is one of the areas that led to the evolution of supramolecular chemistry. A molecular recognition unit is a system whose physiochemical properties change on interaction with a chemical species in such a way as to produce a detectable signal. The molecular recognition unit is analogous to a jigsaw puzzle where the identification of the matching piece is important. Therefore, in molecular recognition the receptor or host interacting with an analyte or guest to form a supramolecular unit is an essential step. A classical example can be obtained from nature. In the case of mammalian cell membrane, the inside of the membrane is selectively permeable to $\mathrm{K}^{+}$and the outside of the membrane to $\mathrm{Na}^{+}$. The selective permeability is due to the presence of receptors present in the sodium and potassium channels, which specifically bind $\mathrm{Na}^{+}$and $\mathrm{K}^{+}$ions and aid the transport of these ions. Another interesting example is the interaction between the base pairs present in the DNA molecule. The DNA molecule contains two strands, which are complementary to each other - the nucleotide sequence in one strand is aligned with a complementary sequence in the opposite strand. A guanine $(\mathrm{G})$ in one strand pairs only with cytosine (C) in the other strand and adenine (A) pairs only with thymine $(\mathrm{T})$ by means of hydrogen bond. This characteristic type of bonding is known as base pairing. Thus, because of the rules of base pairing, one strand can always be used as a template for the synthesis of complementary strand. There are other examples of biological host-guest complexes that include 'enzyme-substrate', 'antigen-antibody' and 'drug-receptor' complexes.

Molecular recognition basically involves hostguest interactions. A site on the guest contains a functional group, which can selectively interact with a complementary functional group of the host. The host-guest interactions can be classified as noncovalent and covalent interactions.

Non-covalent interactions can be further classified into hydrogen bond interaction, electrostatic interaction, interaction between non-polar groups, Pi stacking interactions, chelation of metal ions by ligands and interactions present in molecules with cavities, cages, etc.

4.1a Hydrogen bond interaction: A hydrogen bond (H-bond) donor group of the guest must be positioned near a H-bond acceptor group of the host for tight binding to occur. Organic receptors that bind solely through $\mathrm{H}$-bonds can be considered for molecular recognition. A typical example will be the interaction between phenanthraquinone and di-phenyl urea in aprotic medium.

Figure 2 explains three point $\mathrm{H}$-bond interaction between a flavin and a 2,6-diamidopyridine derivative. ${ }^{26}$ This work, which was carried out by Rotello et al explains how hydrogen bonding and other non-covalent interactions could control flavin reduction.

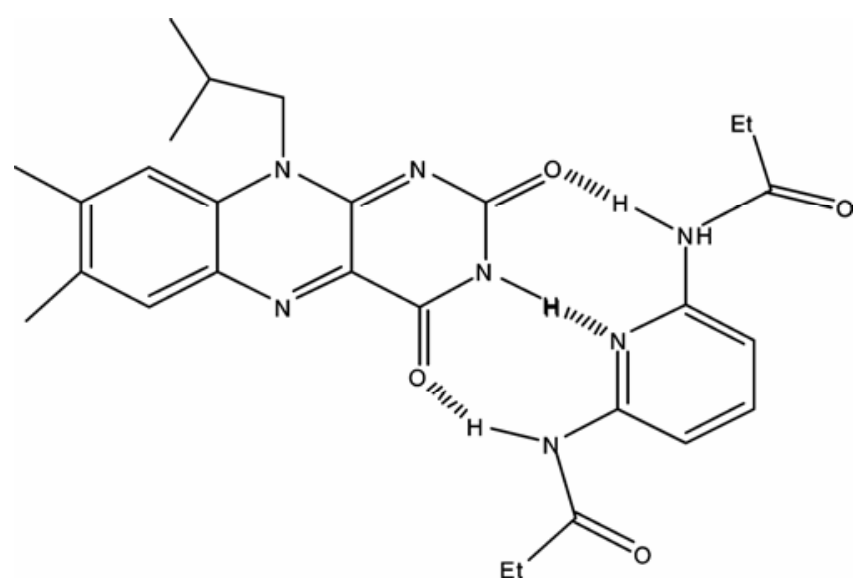

Figure 2. Three point $\mathrm{H}$-bond interaction between a flavin and a 2,6-diamidopyridine derivative. 
Surface modification via 'lock and key' specific self-assembly of polyhedral oligomeric silsequioxane (POSS) derivatives to modified Au surfaces is another example where $\mathrm{H}$-bond interactions are used for molecular recognition. The recognition-mediated modification of thymine terminated SAMs on $\mathrm{Au}$ surfaces using complementary diaminopyridinefunctionalized POSS derivatives has been possible due to H-bonding. ${ }^{27}$

Another approach to stabilizing SAMs is the incorporation of internal H-bond cross-links, mimicking fibrous protein aggregates such as silk and collagen. An interesting account of SAM modified gold substrate stabilized by three layers of internal $\mathrm{H}$ bonds, resulting in a network of lateral crosslinks (cf. figure 3 ) has been reported. ${ }^{28}$ The amide regions of the SAM interact together to form an ordered helical structure.

4.1b Electrostatic interaction: Molecules with polar groups can be manipulated to their maximum polarity by adjusting the $\mathrm{pH}$ and suitably tuned to recognize molecules of opposite polarity. For example, polymer modified, SAM modified electrode surfaces with negatively charged end groups at a particular $\mathrm{pH}$ (cf. figure 4) are used for recognizing the neurotransmitter dopamine that is positively

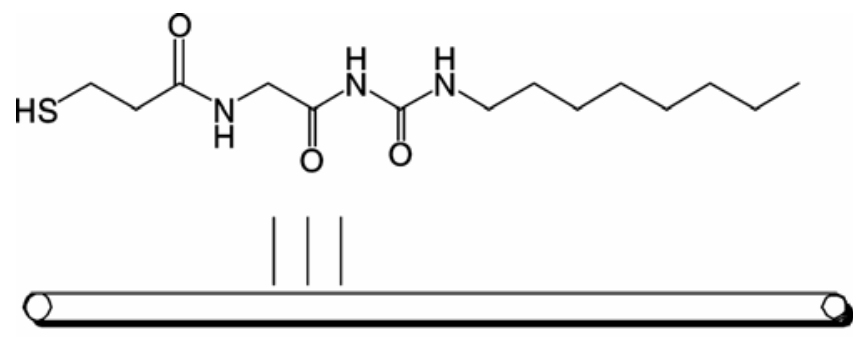

Side view

Figure 3. A thiol molecule stabilized by H-bond through lateral cross-linking.

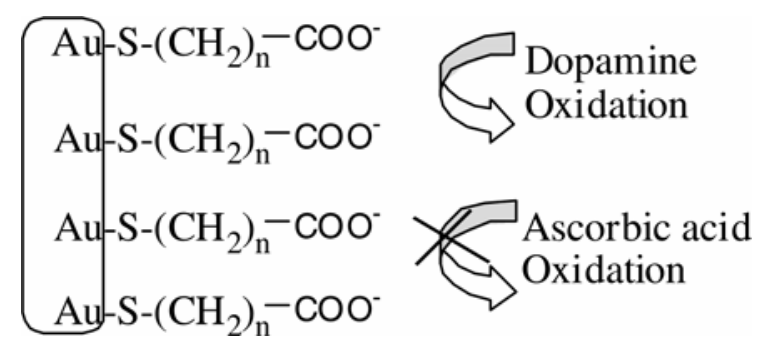

Figure 4. SAM of mercaptoundecanoic acid discriminating between positively charged dopamine and negatively charged ascorbic acid. charged at a neutral $\mathrm{pH}$ in presence of ascorbic acid, which is negatively charged at the same $\mathrm{pH}^{29,30}$

Poly(allylamine) ferrocene has been attached, through electrostatic interaction, to the surface of Au electrode already covered with a SAM of a negatively charged alkane thiol. Thus, the electrode modified with poly(allylamine) ferrocene exhibits excellent electrocatalytic response to the oxidation of ascorbic acid. ${ }^{31}$ Cationic redox polymers containing osmium-bipyridine complexes strongly interact with anionic enzymes, such as glucose oxidase and peroxidases, and electrochemically 'activate' the enzymes. On the basis of these observations, attempts were made to develop an ultrasensitive nucleic acid biosensor. A mixed monolayer of single-stranded oligonucleotide capture probe and 16-mercaptohexadecanoic acid was formed on a gold electrode through self-assembly. Following hybridization with a complementary nucleic acid and glucose oxidase labelled oligonucleotide detection probe, a cationic redox polymer (electrochemical activator) overcoating was applied to the electrode through layer-by-layer electrostatic self-assembly. The formation of an anionic-cationic bilayer brought the glucose oxidase in electrical contact with the redox polymer, making the bilayer an electrocatalyst for the oxidation of glucose. Thus, nucleic acid molecules were quantified amperometrically at femtomolar levels. The effect of experimental variables on the amperometric response was investigated and optimized to maximize the sensitivity and speed up the assay time. A detection limit of $1.0 \mathrm{fmol} / \mathrm{L}$ in $1.0 \mu \mathrm{L}$ droplets and a linear current-concentration relationship up to $800 \mathrm{fmol} / \mathrm{L}$ were attained following $30 \mathrm{~min}$ hybridization. The biosensor was applied to the detection of the $16 \mathrm{~S}$ gene in a mixture of Escherichia coli $16 \mathrm{~S}+32 \mathrm{~S}$ rRNA and a full-length rat housekeeping gene, glyceraldehyde-3-phosphate dehydrogenase (GAPDH), of a RT-PCR product. ${ }^{32}$

4.1c Chelation of metal by ligands: Ligands that can specifically chelate metal ions can be suitably incorporated onto electrode surfaces to fabricate molecular recognition sites for metal ions. SAMs of 2-mercaptobenzimidazole(MBI) on Au surface was reported from our laboratory ${ }^{33}$ towards the recognition of $\mathrm{Hg}(\mathrm{II})$ by stripping voltammetry at low and high $\mathrm{pH}$. The complexation of $\mathrm{Hg}$ (II) ions with MBI molecules occurs at the nitrogen centres (cf. figure 5). The detection limit reported was $0.5 \mathrm{ppm}$ of $\mathrm{Hg}(\mathrm{II})$. 
Selective $\mathrm{K}^{+}$recognition (over $\mathrm{Na}^{+}, \mathrm{Cs}^{+}, \mathrm{Ba}^{2+}$ and $\mathrm{Ca}^{2+}$ ions) has been demonstrated with an acyclic polyether bisthiol. Results suggest that two different binding states of $\mathrm{K}^{+}$exist in the monolayer structure, one that is strongly bound (primary) and another that is weakly bound (secondary). The primary $\mathrm{K}^{+}$ions can be removed irreversibly, but the process is very slow, in contrast to the nearly reversible and fast binding nature of the secondary type. $\mathrm{K}^{+}$does not bind to preformed SAMs, but binds only to monolayers assembled in the presence of $\mathrm{K}^{+}$. Reflectionabsorption infrared spectroscopy (RAIRS) indicates a more compact and oriented monolayer when grown in the presence of $\mathrm{K}^{+}$, providing indirect evidence of the templating process. ${ }^{34}$

Electrochemical sensors for $\mathrm{Cu}$ (II) in environmental samples were prepared by modifying gold electrodes with L-cysteine by self-assembly. The adsorption of L-cysteine on $\mathrm{Au}$ electrodes was studied by electrochemical reductive desorption in $0.5 \mathrm{M}$ $\mathrm{KOH}$, and the interaction of L-cysteine with $\mathrm{Cu}(\mathrm{II})$ was investigated by cyclic voltammetry, chronoamperometry and X-ray photoelectron spectroscopy. At low concentrations, the ratio of L-cysteine to bound $\mathrm{Cu}(\mathrm{II})$ is $2: 1$. At higher concentrations $(0 \cdot 1 \mathrm{M})$ copper reacts with adsorbed cysteine forming copper sulfide on the electrode surface. On a modified Lcysteine gold electrode, Osteryoung square wave voltammetric determination of $\mathrm{Cu}(\mathrm{II})$ with a detection limit below 5 ppb has been demonstrated. ${ }^{35}$

41.d Interaction in molecules with cavities: These are host-guest interactions, which occur in molecules with cavities like cyclodextrins, calixarene, resorcinarene, etc. The cyclodextrins (CDs) are an important class of natural hosts and they do not have any known function in living systems. They are cyclic glucopyranose oligomers and have a clearly defined 'lampshade' shape with a hydrophobic

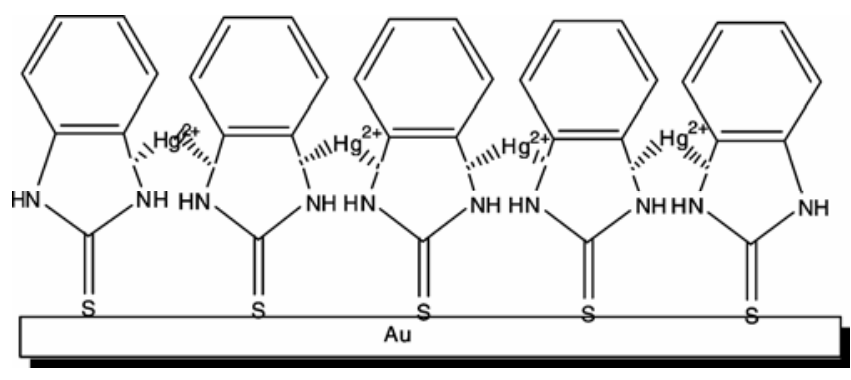

Figure 5. SAM of 2-mercaptobenzimidazole for recognition of $\mathrm{Hg}(\mathrm{II})$ ions. internal cavity and all the hydroxyl groups crowding the two cavity openings. They are soluble in water and form stable inclusion complexes with those nonpolar guests that fit tightly inside the CD cavity. Although the CDs are not electroactive, they do form electroactive inclusion complexes with suitable redox active guests. For instance, $\beta$-CD forms stable $1: 1$ complexes with ferrocene and ferrocene derivatives. Thus, Au surface covered with a SAM of thioctic acid is further modified, through electrostatic interactions, with a layer of positively charged $\beta$-aminocyclodextrin $\left(\beta\right.$-ACD) ${ }^{36}$ The resulting electrode surface, containing $\beta$-ACD as host moieties effectively bind water-soluble ferrocene carboxylate $\left(\mathrm{Fc}-\mathrm{COO}^{-}\right)$species leading to the formation of stable complexes. This demonstrates a typical case of molecular recognition. From our laboratory, it was demonstrated that ferrocene encapsulated hydroxy propyl $\beta$-CD can be used for nitric oxide sensing in sub-micromolar concentrations. The hydroxy propyl $\beta$-CD was tethered to a mercaptoundecanoic acid modified $\mathrm{Au}$ electrode through covalent linkage and was used as a sensing platform for $\mathrm{NO}^{37}$

Cyclophanes are macrocycles made of aromatic ring subunits. Cyclobis(paraquat- $p$-phenylene)-based cyclophanes, first synthesized by Stoddart and coworkers ${ }^{38}$ were themselves found to be redox active. The tetracationic cyclophane has a very rigid structure, with two electron acceptor, 4,4'-bipyridinium subunits lining a cavity which is ideally sized to include aromatic rings. The considerable binding affinity of this cyclophane for $\pi$-donor systems has been extensively utilized by Stoddart's group and others, in the preparation of a large number of catenanes, pseudorotaxanes, and rotaxanes. Unlike CD or calixarene hosts, this cyclophane is electroactive. Usually, the two bipyridinium groups undergo one electron reduction at similar potentials, resulting in a single voltammetric wave. The half-wave potential of this wave is sensitive to complexation, shifting to more negative values on inclusion of a $\pi$ donor guest due to the charge-transfer stabilization of the tetracationic (electron acceptor) form of the host. The host exhibits another cathodic wave at more negative potentials which corresponds to the second one-electron reduction of the bipyridinium groups. This wave can also sometimes be utilized to monitor complexation/decomplexation processes. Kaifer et al synthesized a cyclophane (cf. figure 6), which is structurally similar but contains a disulfide bridge that is expected to serve as its anchoring 
point to Au. Mixed monolayers of cycophane and decanethiol imparted the anticipated molecular recognition properties to the underlying gold electrodes. In the voltammetric experiments with these electrodes, micromolar concentrations of catechol and indole were clearly detected by the shifts measured on the reduction potential of the viologen groups of the immobilized host. ${ }^{36}$

4.1e Covalent interaction: Molecular recognition by covalently bonded systems includes bindings of the receptor molecule to the electrode surface through covalent interactions. The receptor is then ready for sensing the guest molecules. For example, gold substrates modified with thiols and disulfides with suitable terminal groups can be functionalized with enzymes, catalysts and redox species through standard organic reactions. Then the modified surface can be used as a molecular recognition unit.

A layer-by-layer assembly of enzymes and mediators has been formed on electrode surfaces through covalent linkage in our laboratory and they are found to be effective sensing platforms for the detection of sucrose and glucose ${ }^{30,40} \mathrm{On}$ a cleaned $\mathrm{Au}$ surface, cysteamine monolayer was formed and the terminal amino groups were exploited for crosslinking reactions using glutaraldehyde. 1,4-Diamino anthraquinone was used as mediator to which the enzyme glucose oxidase was immobilized.

Cytochrome $\mathrm{C}$ was covalently immobilized using carbodiimide coupling chemistry on a monolayer of 11-mercapto undecanoic acid on gold. The cytochrome $\mathrm{C}$ functionalized monolayer has been characterized by AFM, IR, QCM and direct electrochemistry. Cyclic voltammetric measurements on the chips gave particular redox waves showing characteristics of the surface process. The electroactive protein surface concentration was determined to be $\left.7.2(4.8 \mathrm{pmol} \mathrm{cm})^{-2}\right)$. It was almost consistent with values found in literature, while it was limited to $26 \%$ in magnitude for the QCM data. This was deemed to have arisen from the orientation variation of the surface confined cytochrome $\mathrm{C}$ molecules. $^{41}$

The effect of signal amplification on the bioelectrocatalysis of glucose by dendrimer gold nanocomposite has been recently investigated by us (cf. figure 7). In this work, a layer-by-layer assembly of gold dendrimer nanocomposite and glucose oxidase is demonstrated on $\mathrm{Au}$ surface through covalent linkage using glutaraldehyde. 4.1f Antigen-antibody interactions on SAMs: A new approach to a repeatedly regenerable affinitysensing surface was developed based on the reversible association/dissociation reactions between avidin and biotin analogues. For the affinity surface, a fourth generation poly(amidoamine) dendrimer monolayer was first constructed on MUA SAM on $\mathrm{Au}$. The dendritic surface amine groups then were functionalized with biotin analogues, desthiobiotin (1), or newly synthesized desthiobiotin amidocaproate (2), an extended form of 1 , which shows lower affinity towards avidin. To test the association/dissociation reaction cycles at the affinity surface, avidin adlayer was formed onto the biotin analogue functionalized surface and displaced with free biotin. To trace the step-wise reactions, biotinylated glucose oxidase (b-GOx) as a model enzyme was loaded onto the affinity surface, and cyclic voltammetric measurements were performed by registering the activity of the associated b-GOx. The efficient association/dissociation reaction cycles were registered, especially for the 2-modified electrodes, implying steric hindrance from the ligand length for biospecific interaction. With the optimized affinity-surface construction steps and reaction conditions, continuous association/dissociation

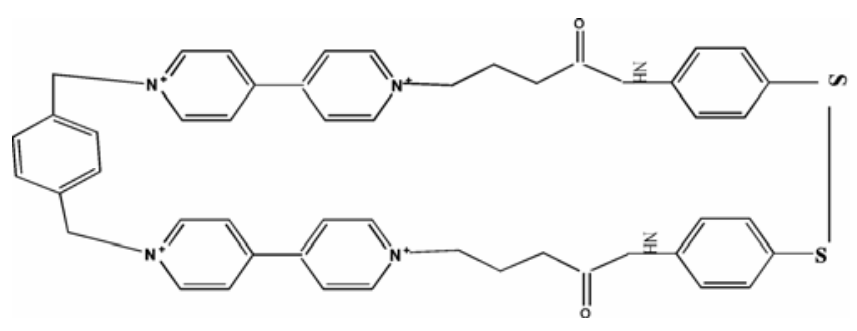

Figure 6. Cyclophane molecule synthesized by Kaifer et al.

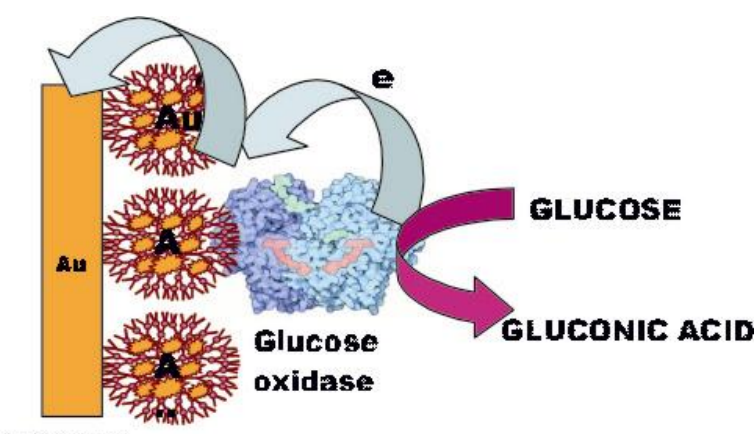

\section{Au-dendrimer}

Figure 7. Assembly of gold dendrimer nanocomposite and glucose oxidase through covalent linkage. Three such layers have been assembled for the analysis of glucose. 


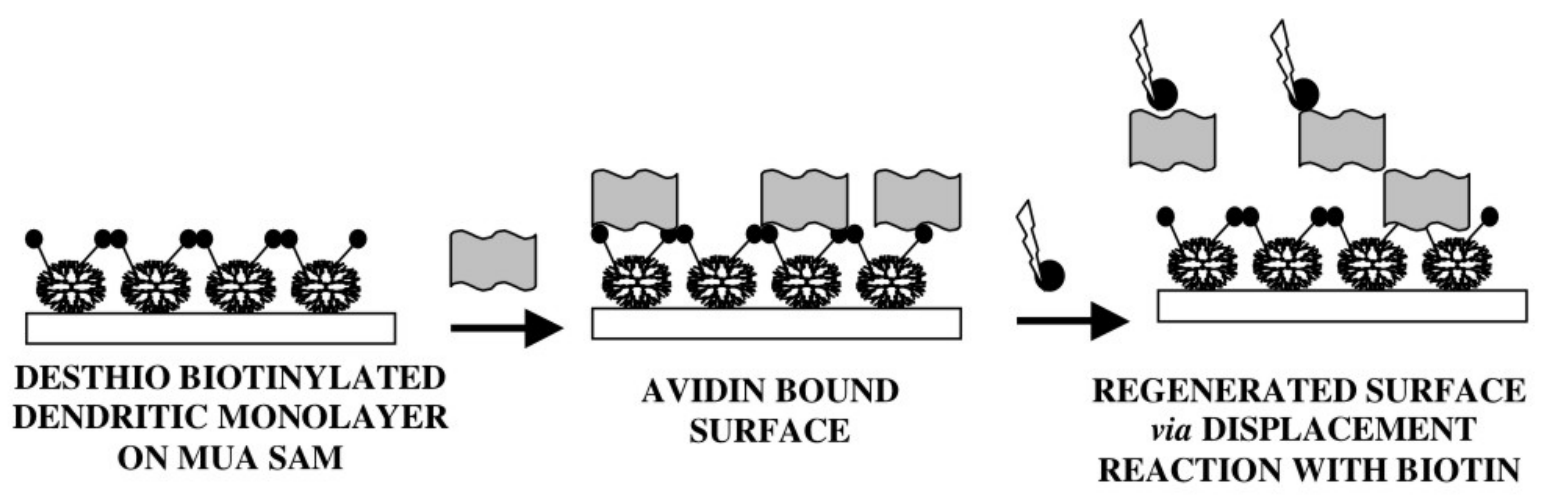

Figure 8. Antigen-antibody interactions on a SAM based platform.

reaction cycles were achieved, resulting in a regenerable affinity surface ${ }^{42}$ (cf. figure 8 ).

\subsection{Controlled crystal morphology on SAM layers}

Control of crystal morphology is critical in many applications of pharmaceutical and food industries. It has been shown recently that SAMs and mixed SAMs of rigid thiols on gold can serve as nucleation planes and modify the morphology of glycine crystals. SAMs and mixed SAMs of 4'-hydroxy-4mercaptobiphenyl, 4-(4-mercaptophenyl)pyridine, and their mixed SAMs with 4'-methyl-4-mercaptobiphenyl were prepared on $\mathrm{Au}(111)$ surfaces and used as templates for the nucleation and growth of glycine crystals. Glycine nucleates in the $\alpha$-glycine structure independent of hydroxy or pyridine surface concentration. The crystallographic planes corresponding to the nucleation surfaces, for the different SAM surfaces under study, were determined by interfacial angle measurements. These differences observed in the crystal morphology are attributed to differences in H-bonding between glycine molecules in the nucleating layer and the SAM surface. As interfacial $\mathrm{H}$-bonding increases, the dipoles of glycine molecules within the crystal become more perpendicular to the SAM surface. The direction of dipoles of glycine molecules that nucleated on a pyridine surface are not as close to the surface normal as those of molecules that nucleated on hydroxyl surface. This implies that the overall $\mathrm{H}$-bonding interactions between the $\mathrm{CO}_{2}^{-}$and $-\mathrm{NH}_{3}^{+}$groups of the glycine and the hydroxyl groups of the SAMs surface are stronger than those between the $-\mathrm{NH}_{3}^{+}$and the pyridine group ${ }^{43}$.

\subsection{Surface patterning using SAMs}

The ability to pattern a surface locally with different molecular monolayers in a well-controlled fashion and at nanoscale resolution has importance for molecular electronics and biotechnology applications, as well as for nanoengineering. A new technique for selectively functionalizing closely spaced gold electrodes of separation below $50 \mathrm{~nm}$ with different thiolated oligonucleotides using a local, selective electrochemical desorption of a molecular protection layer followed by the subsequent adsorption of the oligonucleotides onto the exposed surface is recently reported. ${ }^{44}$ This technique does not rely on the use of a local probe such as an atomic force microscope tip. The surface-bound oligonucleotides retain their unique molecular recognition and self-assembly properties and so functionalize the electrode array.

To sum up, this review essentially indicates how the interfacial architecture can be tailored using strong and weak interactions. Physical adsorption, covalent linkage using standard synthetic organic reactions, hydrogen bonding, electrostatic interactions, host-guest interactions with supramolecules containing nanocavities are the protocols employed to achieve the desired architecture. Over a period of nearly two decades remarkable progress has been made in this field and the applications encompass a number of disciplines like Chemistry, Physics, Biology, Medicine and Nanoscience.

\section{References}

1. Pockels A 1891 Nature 43437

2. Blodgett K 1934 J. Am. Chem. Soc. 56495 
3. Bigelow W C, Pickett D L and Zisman W A $1946 \mathrm{~J}$. Colloid Interface Sci. 1513

4. Ulman A 1991 An introduction to ultrathin organic films: From Langmuir Blodgett to self-assembly (New York: Academic Press)

5. Lee H, Kepley L J, Hong H G and Mallouk T E 1988 J. Am. Chem. Soc. 11061

6. Chen D and Li J 2006 Surface Science Reports 61 445

7. Bain C D, Troughton E B, Tao Y T, Evall J, Whitesides G M and Nuzzo R G $1989 \mathrm{~J}$. Am. Chem. Soc. 111321

8. Bain C D, Evall J and Whitesides G M $1989 \mathrm{~J}$. Am. Chem. Soc. 1117155

9. Troughton E B, Bain C D, Whitesides G M, Nuzzo R G, Allara D L and Porter M D 1988 Langmuir 4 365

10. Bain C D and Whitesides G M $1989 \mathrm{~J}$. Am. Chem. Soc. 1117164

11. Debe M K 1987 Progr. Surf. Sci. 241

12. Hoffmann H, Mayer V, Brunner H and Krischanitz A 1995 Vibrational Spectrosc. 8151

13. Ulman A 1991 ACS Symp. Ser. 447144

14. Futamata M 1995 J. Phys. Chem. 9911901

15. Giuseppe Compagnini, Clelia Galati and Salvatore Pignataro 1999 Phys. Chem. Chem. Phys. 12351

16. Katsuhiko Nishiyama, Shin-ichiro Tahara, Yasutaka Uchida, Shotaro Tanoue and Isao Taniguchi $1999 \mathrm{~J}$. Electroanal. Chem. 47883

17. Bain C D, Troughton E B, Tao Y T, Evall J, Whitesides G M and Nuzzo R G $1989 \mathrm{~J}$. Am. Chem. Soc. 1117155

18. Hungu Kang, Taesung Park, Inchang Choi, Youngil Lee, Eisuke Ito, Masahiko Hara' and Jaegeun Noh 2009 Ultramicroscopy 109111

19. Shinn-Jyh Ding, Bin-Wha Chang, Ching-Chou Wu, Min-Feng Lai and Hsien-Chang Chang 2005 Anal. Chim. Acta $\mathbf{5 4} 43$

20. Porter M D, Bright T B, Allara D L and Chidsey C E D 1987 J. Am. Chem. Soc. 1093559

21. Walczak I M, Popenoe D D, Deinhammer R S, Lamp B D, Chung C and Porter MD 1991 Langmuir 7 2687

22. Sheela Berchmans, Nirmal R G, Prabaharan G, Mishra A K and Yegnaraman V 2006 J. Solid State Electrochem. 10436
23. Xiaoli Cui, Dianlu Jiang, Peng Diao, Junxin Li, Ruting Tong and Xinkui Wang 1999 J. Electroanal. Chem. 4709

24. Ruckenstein E and Li Z F $2005 A d v$. Colloid Interface Sci. 11343

25. Xia Y, Zhao X and Whitesides G M 1996 Microelectron. Eng. 32255

26. Niemz A and Rotello V M 1999 Acc. Chem. Res. 3244

27. Jeoung E, Joseph B Carroll and Rotello V M 2002 Chem. Commun. 1510

28. Clegg R S, Reed S M and Hutchison J E $1998 \mathrm{~J}$. Am. Chem. Soc. 1202486

29. Mary Vergheese T and Sheela B 2004 Mater. Chem. Phys. 83229

30. Mary Vergheese T and Sheela B $2004 \mathrm{~J}$. Electroanal. Chem. 57035

31. Zhang S X, Fu Y Q and Sun C Q 2003 Electroanalysis $\mathbf{1 5} 739$

32. Xie H, Zhang C and Gao Z 2004 Anal. Chem. 76 1611

33. Sheela B, Arivukkodi S and Yegnaraman V 2000 Electrochem. Commun. 2226

34. Bandyopadhyay K, Shu L, Liu H and Echegoyen L 2000 Langmuir 162706

35. Yang W, Justin Gooding J and Brynn Hibbert D J $2001 \mathrm{~J}$. Electroanal. Chem. $\mathbf{5 1 6} 10$

36. Kaifer A E 1999 Acc. Chem. Res. 3262

37. Mary Vergheese T and Sheela B 2006 Electrochimica Acta 52567

38. Odell B, Reddington M V, Slawin A M Z, Spencer N, Stoddart J F and Williams D J 1988 Angew. Chem., Int. Ed. Engl. 271547

39. Sheela B, Sathyajith R and Yegnaraman V 2002 Mater. Chem. Phys. 77390

40. Swaminatha Prabhu K, Sheela B and Yegnaraman V Abs. No. 966, The 198th Meeting of the Electrochemical Society, Phoenix (USA), 22-27 October 2000

41. Nakano K, Yoshitake T, Yamashita Y and Bowden E F 2007 Langmuir 236270

42. Yoon H C, Hong M and Kim H 2001 Langmuir 17 1234

43. Kang J F, Zaccaro J, Ulman A and Myerson A 2000 Langmuir 163791

44. Walti C, Wirtz R, André Germishuizen W, Bailey D M D, Pepper M, Middelberg A P J and Giles Davies A 2003 Langmuir 19981 